\title{
On super free fall
}

\author{
A. Torres ${ }^{1}$, A. Medina ${ }^{1}$, F. J. Higuera ${ }^{2}$ and P. D. Weidman ${ }^{3} \dagger$ \\ ${ }^{1}$ ESIME UA, Instituto Politécnico Nacional, Avenida de las Granjas 180, C.P. 02250, México D.F., \\ Mexico \\ ${ }^{2}$ Escuela Técnica Superior de Ingenieros Aeronáuticos, Plaza del Cardenal Cisneros 3, 28040 Madrid, \\ Spain \\ ${ }^{3}$ Department of Mechanical Engineering, University of Colorado, Boulder, CO 80309, USA
}

Villermaux \& Pomeau (J. Fluid Mech., vol. 642, 2010, p. 147) analysed the motion of the interface of an inviscid liquid column released from rest in a vertical tube whose area expands gradually downwards, with application to an inverted conical container for which experimental measurements were carried out. An error in the analysis is found and corrected in the present investigation, which provides the new governing equation for the super-accelerated interface motion down gradually varying tubes in general, and integrated results for interface trajectories, velocities and accelerations down a conical tube in particular. Interestingly, the error does not affect any of the conclusions given in the 2010 paper. Further new results are reported here such as the equation governing the centre of mass and proof that the end point acceleration is exactly that of gravity.

Key words: interfacial flows (free surface)

\section{Introduction}

The super free fall work of Villermaux \& Pomeau (2010, hereafter often simply $\mathrm{V} \& \mathrm{P}$ ) is revisited. The problem is as follows. Taking $z$ as the upward coordinate, a tube of cross-sectional area $a(z)$ slowly increasing downwards is capped at the bottom, where $z=0$, and filled with a low-viscosity liquid to height $z=h(0)$ with interface exposed to the atmosphere. At $t=0$ the cap vanishes and liquid flows out the end of the tube into the ambient atmosphere.

V\&P rederived the equation of Paterson (1983):

$$
\left(a(h) \int_{0}^{h} \frac{\mathrm{d} z}{a(z)}\right) \ddot{h}+\frac{1}{2}\left(1-\frac{a(h)^{2}}{a(0)^{2}}\right) \dot{h}^{2}=-g h .
$$

Here $g$ is gravity and $z=h(t)$ is the height of the downward-moving upper free surface.

The equation reported by Paterson (1983) was formulated for unsteady potential flow in a tube composed of a long section of uniform cross-sectional area $A_{1}$, which at some low level gently varies to fixed cross-sectional area $A_{0}$. Paterson assumed that the level of the upper interface always remained in the section of uniform area $A_{1}$, but this restriction can be easily removed.

$†$ Email address for correspondence: weidman@ colorado.edu 
The equation for the area-averaged vertical velocity in the tube used by both Paterson (1983) and Villermaux \& Pomeau (2010) is $w(z, t)=a(h) \dot{h} / a(z)$, where an overdot represents differentiation with respect to time. The evolution equation for the height of the liquid is obtained by carrying this velocity to the momentum equation for an inviscid liquid, $\partial w / \partial t+\partial\left(w^{2} / 2+p / \rho+g z\right) / \partial z=0$, and integrating along the tube from $z=0$ to $z=h$. The time derivative of $w$ required in the momentum equation is

$$
\frac{\partial w}{\partial t}=\frac{a(h)}{a(z)} \ddot{h}+\frac{1}{a(z)} \frac{\mathrm{d} a(h)}{\mathrm{d} h} \dot{h}^{2},
$$

and the momentum equation integrated along the tube gives the generalized Paterson equation:

$$
\left(a(h) \int_{0}^{h} \frac{\mathrm{d} z}{a(z)}\right) \ddot{h}+\frac{\mathrm{d} a(h)}{\mathrm{d} h} \int_{0}^{h} \frac{\mathrm{d} z}{a(z)} \dot{h}^{2}+\frac{1}{2}\left(1-\frac{a(h)^{2}}{a(0)^{2}}\right) \dot{h}^{2}=-g h .
$$

The second term on the right-hand side of (1.2), which leads to the middle term on the left-hand side of (1.3), was neglected by V\&P. This term is necessary to account for the acceleration in variable-area sections of a tube and, for that reason, we denote it as the variable-area temporal acceleration, or VAT acceleration for short.

A rough order-of-magnitude estimate shows that the coefficient of $\dot{h}^{2}$ in the middle term on the left-hand side of $(1.3)$ is $(\mathrm{d} a / \mathrm{d} h) \int_{0}^{h} \mathrm{~d} z / a=O\left[\left(\Delta a / h_{c}\right)\left(h_{c} / a_{c}\right)\right]=$ $O\left(\Delta a / a_{c}\right)$, where $a_{c}$ and $\Delta a$ are characteristic values of the cross-sectional area of the tube and of its variation along the tube, say from the bottom to the initial height of the interface, and $h_{c}$ is a characteristic value of that interface height. Therefore, the second and third terms on the left-hand side of (1.3) are both of the same order when the variation of $a$ in the region of the tube containing the liquid interface is of the order of $a$ itself.

Equation (1.3) takes a simple form when the tube is a vertical cone of height $L$ with a small opening angle, $\alpha \ll 1$. In this case $a(z)=\pi \alpha^{2}(L-z)^{2}$, giving $\int_{0}^{h} \mathrm{~d} z / a=(h / L)(L-h) / a(h)$ and, upon introducing the dimensionless variables

$$
\xi=1-\frac{h}{L} \quad \text { and } \quad \tau=\sqrt{\frac{g}{L}} t,
$$

the governing initial-value problem

$$
\ddot{\xi}=\frac{1}{\xi}+\frac{1}{2}\left[1+\xi+\xi^{2}-\frac{3}{\xi}\right] \dot{\xi}^{2}, \quad \xi(0)=\xi_{0}, \quad \dot{\xi}(0)=0
$$

is obtained. Initial liquid heights $0<h(0)<L$ correspond to initial values $1>\xi_{0}>0$.

Using Paterson's 1983 equation (1.1), Villermaux \& Pomeau (2010) obtained fundamental results for the motion of a liquid surface descending in an expanding cone. These include determination of the initial acceleration and pressure gradient at the free surface, and demonstration that the acceleration of the centre of mass is initially less than gravity. $\mathrm{V} \& \mathrm{P}$ also presented an analysis of how the superacceleration triggers a Rayleigh instability of the free surface leading to the formation of a nipple and a drop, and the relevance of this to wave breaking. We note at the outset that none of these results are affected by neglect of the VAT acceleration. However, as will be shown in the sequel, the position, velocity and acceleration of the interface change significantly when this acceleration is retained. 
Following Villermaux \& Pomeau (2010), the goal of the present investigation is to determine the motion of the upper free surface in a slender, downward-opening cone. In addition, we derive the equation governing the motion of the centre of the decreasing liquid mass in the tube and prove that the accelerations of the interface and centre of mass both tend to $-g$ at the terminal moment when all liquid is exhausted from the tube.

We begin in $\$ 2$ with new analytic results and numerical integrations for the interface position, velocity and acceleration. This section ends with a detailed study of the influence of the VAT acceleration on interface trajectory and acceleration. The results of a laboratory experiment are compared with theory in $\S 3$, and a discussion and concluding remarks are given in $\S 4$.

\section{Analysis and numerical results}

Equation (1.5) for the dimensionless position of the liquid interface $\xi(\tau)$ is autonomous and thus a reduction of order is ensured. In this manner a linear firstorder equation for $\dot{\xi}^{2}$ as a function $\xi$ is obtained. Solution of this equation with the condition $\dot{\xi}^{2}=0$ at $\xi=\xi_{0}$ gives

$$
\dot{\xi}=\left[2 f(\xi) \int_{\xi_{0}}^{\xi} \frac{\mathrm{d} \eta}{\eta f(\eta)}\right]^{1 / 2}, \quad f(\xi)=\frac{1}{\xi^{3}} \exp \left(\xi+\frac{\xi^{2}}{2}+\frac{\xi^{3}}{3}\right)
$$

which can be integrated again to obtain $\tau=\tau\left(\xi, \xi_{0}\right)$. However, numerical integration of (1.5) using a fourth-order Runge-Kutta method is more appropriate for our purposes here. Before presenting numerical solutions in $\$ 2.4$, further analysis of the super free fall motion will now be given.

\subsection{Singular behaviour for $\xi_{0}=0$}

The evolution of the free surface displays two distinct stages for small values of $\xi_{0}$. The velocity first increases from 0 to $(2 / 3)^{1 / 2}$ in a short stage $\tau=O\left(\xi_{0}\right)$ where the acceleration is $\ddot{\xi}=O\left(\xi_{0}^{-1}\right)$. Equation (2.1) can be simplified in this stage using the approximation $f(\xi) \approx \xi^{-3}$, which gives $\dot{\xi}=(2 / 3)^{1 / 2}\left(1-\xi_{0}^{3} / \xi^{3}\right)^{1 / 2}$ and, upon further integration,

$$
\tau=(3 / 2)^{1 / 2} \xi_{0} I\left(\xi / \xi_{0}\right), \quad I\left(\xi / \xi_{0}\right)=\int_{1}^{\xi / \xi_{0}} \frac{\chi^{3 / 2} \mathrm{~d} \chi}{\left(\chi^{3}-1\right)^{1 / 2}} .
$$

This is followed by a longer stage $\tau=O(1)$ where the evolution is given by solution of the equation in (1.5) with the modified conditions $\xi(0)=0$ and $\dot{\xi}(0)=(2 / 3)^{1 / 2}$, obtained from asymptotic matching with the first stage in the limit $\xi_{0} \rightarrow 0$. The terminal time $\tau_{f}$ at which $\xi\left(\tau_{f}\right)=1$ is $\tau_{f} \approx 1.083$ for this singular case.

\subsection{Centre-of-mass motion}

The height $h_{c m}$ and velocity $w_{c m}$ of the centre of mass of the liquid in the tube are given by

$$
V h_{c m}=\int_{V} z \mathrm{~d} \Omega=\int_{0}^{h} z a(z) \mathrm{d} z, \quad V w_{c m}=\int_{V} w \mathrm{~d} \Omega=\int_{0}^{h} w a(z) \mathrm{d} z=a(h) h \dot{h}, \quad(2.3 a, b)
$$

where $V$ is the volume of liquid in the tube. The last equality, obtained from the equation of continuity $a(z) w(z, t)=a(h) \dot{h}$, was found by Villermaux \& Pomeau (2010). 
The acceleration of the centre of mass is not simply $\mathrm{d} w_{c m} / \mathrm{d} t$ because $V$ is not a constant. To compute this acceleration, here denoted as $a_{c m}$, we consider the material volume $V_{f}$ enclosing the liquid mass $m$ that is in the tube at a given instant $t$. This mass is a constant. As time goes on, part of the liquid flows out of the tube, but not out of the material volume $V_{f}$. The velocity of the centre of mass of the liquid in $V_{f}$ is given by $m w_{c m}=\int_{V_{f}} \rho w \mathrm{~d} \Omega$ at time $t$, and since $m$ is a constant,

$$
m a_{c m}=\frac{\mathrm{d}}{\mathrm{d} t} \int_{V_{f}} \rho w \mathrm{~d} \Omega=\frac{\mathrm{d}}{\mathrm{d} t} \int_{V} \rho w \mathrm{~d} \Omega-\int_{\Sigma_{b}} \rho w^{2} \mathrm{~d} \sigma,
$$

where $\Sigma_{b}$ is the cross-section at the bottom of the tube and the last equality follows from Reynolds' transport theorem. Evaluation of the volume integral on the right-hand side of (2.4) gives $\rho a(h) h \dot{h}$ and the surface integral is $\rho a(0) w(0, t)^{2}=\rho a(h)^{2} \dot{h}^{2} / a(0)$, so that

$$
m a_{c m}=\frac{\mathrm{d}}{\mathrm{d} t}(\rho a(h) h \dot{h})-\rho \frac{a(h)^{2}}{a(0)} \dot{h}^{2}=\rho a(h) h \ddot{h}+\rho\left[a(h)+h \frac{\mathrm{d} a(h)}{\mathrm{d} h}-\frac{a(h)^{2}}{a(0)}\right] \dot{h}^{2} .
$$

Using (1.3) to eliminate $\ddot{h}$ we find

$$
\begin{aligned}
a_{c m}= & -\frac{g h^{2}}{\left(\int_{0}^{h} a \mathrm{~d} z\right)\left(\int_{0}^{h} \mathrm{~d} z / a\right)} \\
& -\frac{1-a(h) / a(0)}{\int_{0}^{h} a \mathrm{~d} z}\left[\frac{1}{2}\left(1+\frac{a(h)}{a(0)}\right) \frac{h}{\int_{0}^{h} \mathrm{~d} z / a}-a(h)\right] \dot{h}^{2} .
\end{aligned}
$$

Since initially $\dot{h}=0$, it is clear that the first term of (2.6) gives the early-time centreof-mass acceleration, in agreement with the result reported as (3.17) in Villermaux \& Pomeau (2010).

\subsection{Terminal acceleration}

A simple analysis gives the interface acceleration at the terminal time $t_{f}$, just when the liquid in the tube is exhausted. This is achieved using the integral form of the vertical momentum equation for the liquid in the tube (see e.g. Batchelor 1967, p. 138)

$$
m a_{c m}=-\int_{\Sigma_{w}} p n_{z} \mathrm{~d} \sigma-\int_{V} \rho g \mathrm{~d} \Omega,
$$

where $m a_{c m}$ is given by (2.5), $p$ is the pressure of the liquid referred to the atmospheric pressure outside the tube, $\Sigma_{w}$ is the surface of the tube wall in contact with the liquid, and $n_{z}$ is the vertical component of the unit normal to this surface ( $n_{z}=\alpha$ for a conical tube). The factor in square brackets in (2.5) is of $O\left(h^{2}\right)$ for small $h$, as can be seen by Taylor-expanding $a(h)$. The first term on the right-hand side of (2.7) is the force the tube wall exerts on the liquid. Since the acceleration of the liquid is finite, as must be the pressure gradient inside the liquid, $\mathrm{d} p / \mathrm{d} z=O(\rho g)<\infty$, so that $p=O(\rho g h)$ and therefore $-\int_{\Sigma_{w}} p n_{z} \mathrm{~d} \sigma=O\left(\rho g n_{z} a(0)^{1 / 2} h^{2}\right)$. Finally, the body force for small $h$ is $-\int_{V} \rho g \mathrm{~d} \Omega \approx-\rho g a(0) h$. Therefore the dominant terms in (2.7) are proportional to $h$ and the $h \rightarrow 0$ balance gives $\ddot{h}\left(t_{f}\right)=-g$. 


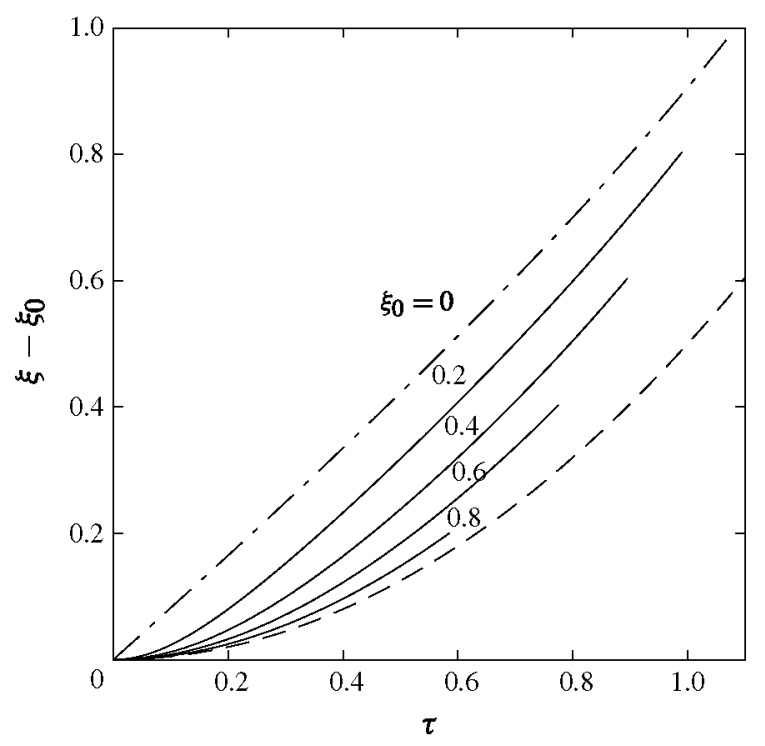

FIGURE 1. Evolution of the interface position $\xi-\xi_{0}$ for selected initial values $\xi_{0}$. The singular perturbation position for $\xi_{0}=0$ is shown by the dot-dashed line and the pure free fall trajectory given in (2.8) is displayed by the dashed line.

A similar small- $h$ analysis of the centre-of-mass acceleration given in (2.6) can be used to show that $a_{c m}\left(t_{f}\right)=-g$. Thus both the free surface and the centre of mass experience Earth's gravity at the moment when all liquid is discharged from the tube. These results are independent of the cross-sectional variation of the tube area as long as this area is a slowly varying function of $z$.

\subsection{Numerical results}

Numerical integrations of the initial-value problem (1.5) are now presented for initial values $\xi_{0}=0.2,0.4,0.6,0.8$. Integrations are carried out to the terminal time $\tau_{f}$ when all liquid is exhausted from the tube, corresponding to $\xi=1$. Interface positions, velocities and accelerations will be compared with pure free fall results, which, in non-dimensional coordinates, are given by

$$
\xi=\frac{\tau^{2}}{2}+\xi_{0}, \quad \dot{\xi}=\tau, \quad \ddot{\xi}=1 .
$$

Figure 1 shows the time evolution of the free surface, $\xi-\xi_{0}$, at the selected values $\xi_{0}$. Each curve ends at the terminal time $\tau_{f}$ defined by $\xi\left(\tau_{f}\right)=1$. The results show that the interface in the slowly expanding cone falls more rapidly than fluid in pure free fall shown by the dashed line. The outer singular solution for $\xi_{0}=0$ is also displayed as the dot-dashed line. It is evident that trajectories for smaller initial values $\xi_{0}$ cover distances faster than for larger $\xi_{0}$.

The time evolution of interface velocities plotted up to the terminal time $\tau_{f}$ in figure 2 confirms, at each value $\xi_{0}$, that the free surface velocities are always greater than those for pure free fall indicated by the dashed line. Also included in this figure is the outer singular solution for $\xi_{0}=0$ plotted as the dot-dashed line. Higher liquid fills (smaller $\xi_{0}$ ) exhibit higher velocities at the early stages, but the situation may 


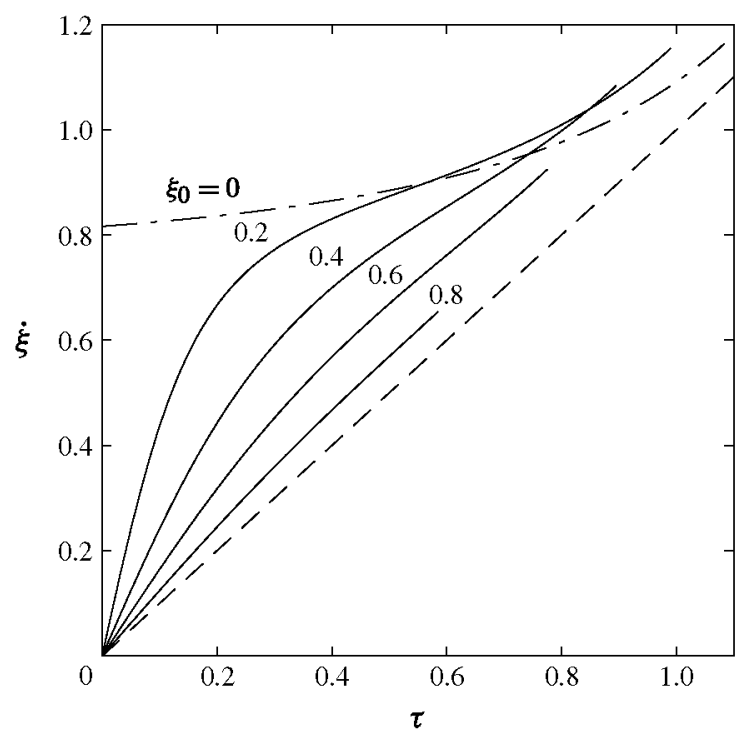

FIGURE 2. Evolution of the interface velocity $\dot{\xi}$ for selected initial values $\xi_{0}$. The singular perturbation velocity for $\xi_{0}=0$ is shown by the dot-dashed line and the pure free fall velocity given in (2.8) is displayed by the dashed line.

reverse at later times, as illustrated by the intersection of the curve for $\xi_{0}=0.2$ with that for $\xi_{0}=0.4$ at $\tau \approx 0.87$.

This curious feature may be understood by comparing the evolution of free surface accelerations presented in figure 3. Here again the constant pure gravity acceleration is displayed as the dashed line and the outer singular solution for $\xi_{0}=0$ is plotted as the dot-dashed line. All curves in figure 3 begin with a super-gravitational acceleration equal to $1 / \xi_{0}$, cross the dashed free fall line to sub-gravitational accelerations, and then turn back to terminate at exactly gravitational acceleration as required (cf. $\$ 2.3$ ). In particular, it is seen that, while the initial acceleration for $\xi_{0}=0.2$ is high $(\ddot{\xi}=5)$, it attains significant sub-gravitational acceleration over a major portion of its evolution. Since the range and intensity of the sub-gravitational acceleration for $\xi_{0}=0.4$ are small compared to those for $\xi_{0}=0.2$, the velocity for $\xi_{0}=0.4$ in figure 2 eventually surpasses that for $\xi_{0}=0.2$ before the $\xi_{0}=0.4$ curve attains its terminal time.

We conclude this section by showing the variation of $\tau_{f}$ with $\xi_{0}$ in figure 4 . The numerical results plotted by the solid dots are connected by straight lines, the lowest and highest values of $\xi_{0}$ being 0.01 and 0.995 , respectively. The small terminal times have been extrapolated to the obvious result $\tau_{f}=0$ at $\xi_{0}=1$. The large terminal times are seen to merge smoothly to the value $\tau_{f} \approx 1.083$ at $\xi_{0}=0$ obtained from the singular analysis given in $\$ 2.1$.

\subsection{Influence of the VAT acceleration}

The goal in this section is to compare results obtained from (1.1) and (1.3) for conditions of the inverted cone experiment presented in table 1 of Villermaux \& Pomeau (2010) and, at the suggestion of a referee, to assess the relative importance of the acceleration terms proportional to $\dot{h}^{2}$. The exit radius is $R(0)$ and the initial height of the upper free surface is $h(0)$, where the radius is $R(h(0))$. The cone for their 


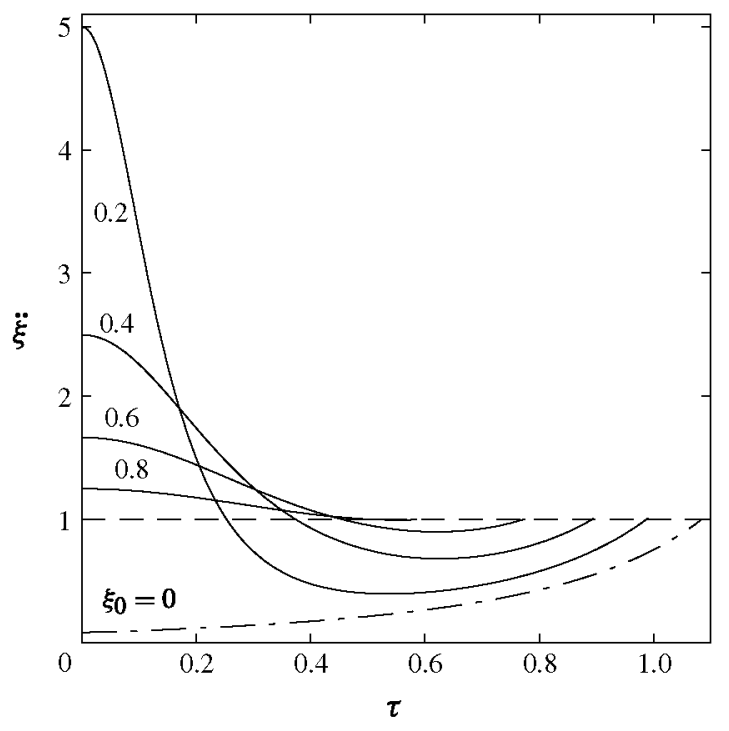

FIGURE 3. Evolution of the interface acceleration $\ddot{\xi}$ for selected initial values $\xi_{0}$. The singular perturbation acceleration for $\xi_{0}=0$ is shown by the dot-dashed line and the pure free fall acceleration given in (2.8) is displayed by the dashed line.

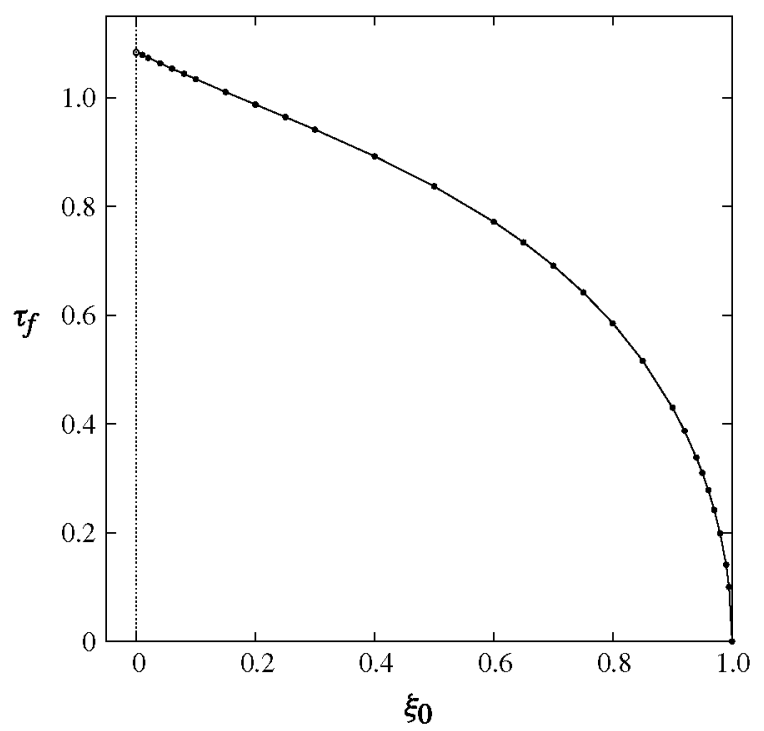

FIGURE 4. Emptying time $\tau_{f}$ as a function of $\xi_{0}$. The computed values shown as solid dots are connected by straight line sections and the result $\tau_{f} \approx 1.083$ obtained from the singular analysis for $\xi_{0}=0$ is shown by the open circle.

experiment has the geometrical properties

$$
\tan \alpha=0.03, \quad R(0)=0.033 \mathrm{~m}, \quad \beta=1-\frac{R(h(0))}{R(0)}=0.33,
$$




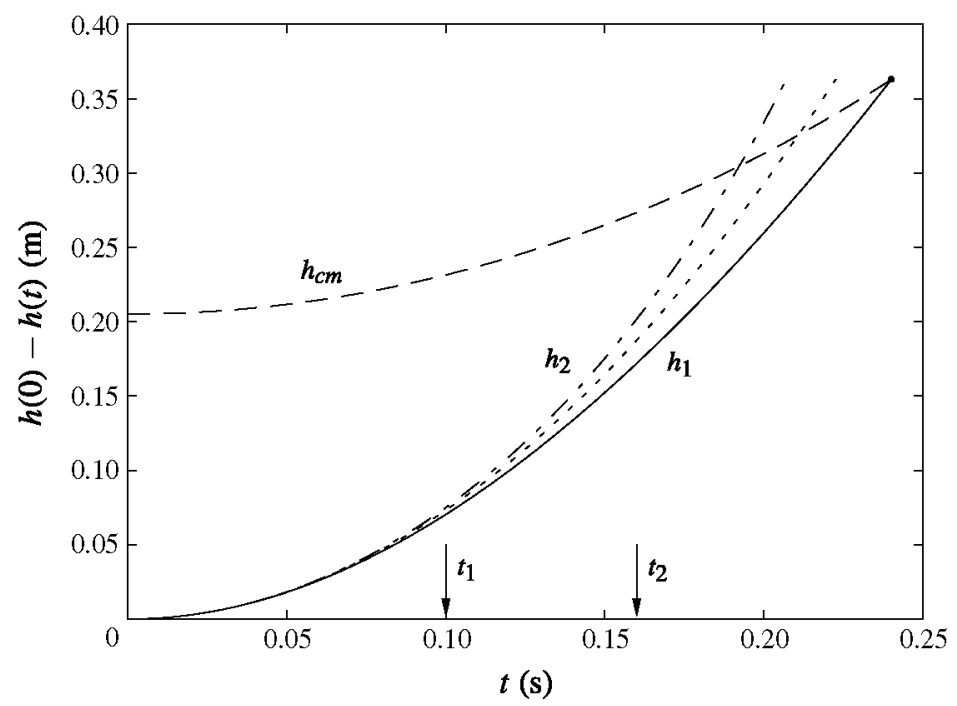

FIGURE 5. Trajectories $h_{1}$ and $h_{2}$ computed from (2.10) for conditions of the V\&P experiment with and without the VAT acceleration straddle the trajectory obtained by evolving the motion at constant super-acceleration $\ddot{h}(0)$ plotted as the short dashed line. Also included is the centre-of-mass trajectory $h_{c m}$. The arrow $t_{2}$ marks the upper limit of the V\&P experimental data and the arrow $t_{1}$ is the upper limit for which V\&P compared their data to the $\ddot{h}(0)$ trajectory in a $\log -\log$ plot.

which are used to determine the initial fill height $h(0)=0.363 \mathrm{~m}$ and the height $L=2.75 \mathrm{~m}$ to the apex. The dimensional equation governing interface motion obtained from (1.3) may be written as

$$
h(1-k h) \ddot{h}-2 k h \dot{h}^{2}+\frac{1}{2}\left(1-(1-k h)^{4}\right) \dot{h}^{2}=-g h,
$$

wherein $k=1 / L$. The term $-2 k h \dot{h}^{2}$ in $(2.10)$ is the contribution from the VAT acceleration. Integrations of this equation with and without the VAT acceleration have been carried out for the experimental value $k=0.9091 \mathrm{~m}^{-1}$ using $g=9.81 \mathrm{~m} \mathrm{~s}^{-2}$. We denote the trajectories and accelerations obtained from (2.10) as $h_{1}$ and $\ddot{h}_{1}$ and those with the VAT acceleration missing as $h_{2}$ and $\ddot{h}_{2}$. The $h_{1}$ and $h_{2}$ trajectories plotted in figure 5 straddle the trajectory obtained assuming that the acceleration is uniform, fixed at its initial value $\ddot{h}(0)=-g /(1-\beta)=-14.642 \mathrm{~m} \mathrm{~s}^{-2}$ shown as the short dashed line. The computed terminal time $t_{f}$ is shortest for the $h_{2}$ trajectory $(0.207 \mathrm{~s})$, increases for the constant $\ddot{h}(0)$ trajectory $(0.223 \mathrm{~s})$, and is longest for the $h_{1}$ trajectory $(0.240 \mathrm{~s})$. The vertical arrow at $t_{2}=0.16 \mathrm{~s}$ marks the upper limit over which Villermaux \& Pomeau (2010) reported data, and the arrow at $t_{1}=0.10 \mathrm{~s}$ is the upper limit for which these data were compared to the constant- $\ddot{h}(0)$ trajectory. The centre-of-mass trajectory $h_{c m}$ and its initial value for a cone computed from $(2.3 a)$,

$$
h_{c m}(t)=\frac{h}{4}\left(\frac{6-8 k h+3 k^{2} h^{2}}{3-3 k h+k^{2} h^{2}}\right), \quad h_{c m}(0)=\frac{h(0)}{4}\left(\frac{6-8 \beta+3 \beta^{2}}{3-3 \beta+\beta^{2}}\right),
$$

are also plotted in figure 5. 


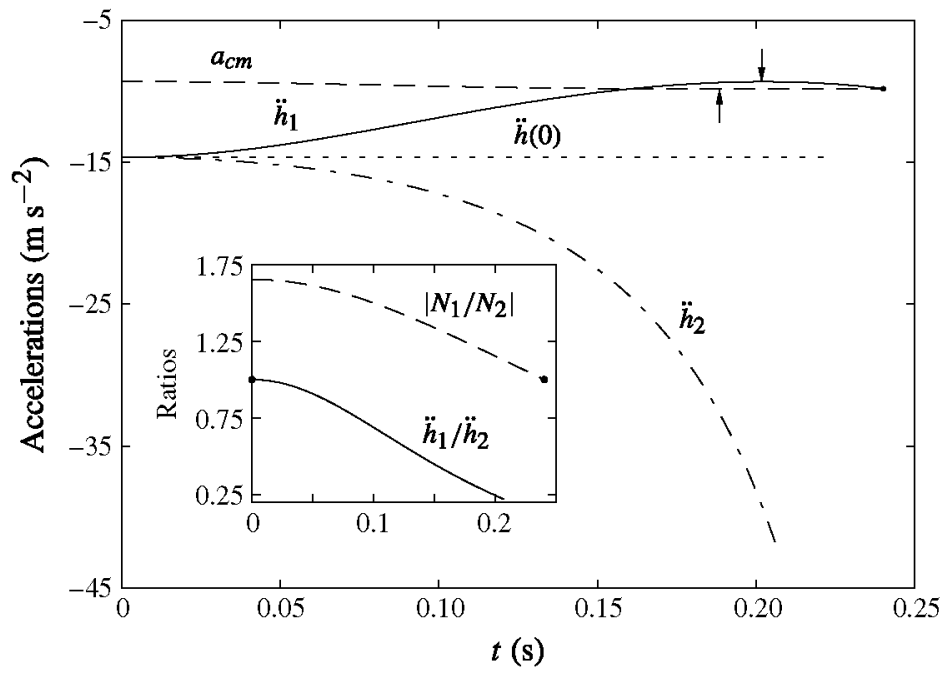

FIGURE 6. Accelerations $\ddot{h}_{1}$ and $\ddot{h}_{2}$ computed from (2.10) for the V\&P experiment with and without the VAT acceleration straddle the initial acceleration $\ddot{h}(0)$. Also shown is the centre-of-mass acceleration $a_{c m}$. Arrows mark extrema points in the evolution of $a_{c m}$ and $\ddot{h}_{1}$. The inset shows the ratio $\ddot{h}_{1} / \ddot{h}_{2}$ as well as the ratio $\left|N_{1} / N_{2}\right|$ of the nonlinear terms defined in (2.13).

Several things may be observed from the trajectories plotted in figure 5. First, the (shifted) centre-of-mass trajectory starts with zero slope and increases to merge, as required from the analysis in $\S 2.3$, with the $h_{1}$ trajectory at the terminal time. Second, the percentage deviation between the $h_{1}$ and $h_{2}$ trajectories increases uniformly over all time and attains the value $6.6 \%$ at $t_{1}$. Since the constant-acceleration trajectory lies even closer to $h_{1}$ than does $h_{2}$, it is not surprising that Villermaux \& Pomeau's 2010 experimental data compare very favourably with the constant-acceleration trajectory in the region $0.01 \mathrm{~s} \leqslant t \leqslant t_{1}$ over which their comparison was made. Consequently, for this $0.363 \mathrm{~m}$ liquid fill level, one may take the $6.6 \%$ deviation at $t=t_{1}=0.10 \mathrm{~s}$ as the short-time limit for which the data of V\&P are accurate; note at this point that the liquid has descended only $20 \%$ of its distance to the bottom of the tube. At later stages in the free fall, interface deviations increase to $17 \%$ at $t_{2}$ and on to a maximum value slightly over $31 \%$ at the largest time $t_{f}=0.207 \mathrm{~s}$ for which this comparison can be made.

The third observation concerns the remark of Villermaux \& Pomeau (2010) that the nonlinear term proportional to $\dot{h}^{2}$ in (1.1) increases the super-acceleration relative to the initial acceleration at late stages. The acceleration $\ddot{h}_{2}$ plotted in figure 6 (introduced in the following paragraph) indeed confirms this result. V\&P also noted that their experimental data exhibit a slowdown probably '... attributable to the friction of the engulfed air at the upper tube opening ...'. Figure 5 shows that the 'slowdown' is a real effect predicted by the $h_{1}$ trajectory that includes the VAT acceleration and, consequently, the above caveat about air entrainment is not necessary.

Now consider the evolution of interface accelerations $\ddot{h}_{1}$ and $\ddot{h}_{2}$. These accelerations plotted in figure 6 are seen to be respectively higher and lower than the initial acceleration value $\ddot{h}(0)$ plotted as the short dashed line. In addition, the centre-of-mass 
acceleration and its initial value determined from (2.6) for a cone,

$$
\left.\begin{array}{l}
a_{c m}(t)=-\frac{1-k h}{1-k h+\frac{1}{3} k^{2} h^{2}}\left[g+k^{3} h^{2}\left(1+\frac{1}{2} k h\right) \dot{h}^{2}\right], \\
a_{c m}(0)=-\frac{(1-\beta) g}{1-\beta+\frac{1}{3} \beta^{2}},
\end{array}\right\}
$$

are plotted as the long dashed line. For the Villermaux \& Pomeau (2010) value $\beta=0.33, a_{c m}(0)=-9.306 \mathrm{~m} \mathrm{~s}^{-2}$, only $5 \%$ smaller in absolute value than $g$. Both $\ddot{h}_{1}$ and $a_{c m}$ achieve an extremum at late stages. The minimum value $a_{c m}=-9.828 \mathrm{~m} \mathrm{~s}^{-2}$ occurs at $t=0.189 \mathrm{~s}$ whilst the maximum value $\ddot{h}_{1}=-9.317 \mathrm{~m} \mathrm{~s}^{-2}$ occurs at $t=0.203 \mathrm{~s}$. In the inset of figure 6 the evolution of the ratio $\ddot{h}_{1} / \ddot{h}_{2}$ is plotted beginning with its value of unity at $t=0$. Since $\ddot{h}_{1} / \ddot{h}_{2} \leqslant 1$, the trajectory $h_{1}$ with the VAT acceleration markedly slows down relative to the trajectory $h_{2}$ with the VAT acceleration omitted, as observed in figure 5.

Finally, we compare the relative magnitude of the nonlinear terms in (2.10) proportional to $\dot{h}^{2}$. For the cone experiment of Villermaux \& Pomeau (2010), these are denoted

$$
N_{1}(t)=-2 k h \dot{h}^{2}, \quad N_{2}(t)=\frac{1}{2}\left(1-(1-k h)^{4}\right) \dot{h}^{2} .
$$

The ratio $\left|N_{1} / N_{2}\right|$, shown in the inset of figure 6 as the dashed line, decreases from its initial value 1.654 to the dot at the terminal time $t_{f}=0.240 \mathrm{~s}$. One must bear in mind, however, that $N_{1}<0$ and $N_{2}>0$ for all $0<t<t_{f}$ and thus the VAT term $N_{1}$ acts opposite to $N_{2}$. It is recognized that both these terms are initially zero. Nevertheless, since $\left|N_{1} / N_{2}\right| \geqslant 1$, the influence of the nonlinear VAT term dominates for all $t>0$.

\section{Experiments}

A series of experiments on the super-accelerated flow down inverted cones were undertaken using three conical glass tubes. Though more than five experiments were performed, and observations of the nipple formation were recorded, for the sake of brevity we report here only one experimental result. The tube for this experiment had lower diameter $D_{1}=2.5 \mathrm{~cm}$, upper diameter $D_{2}=4.7 \mathrm{~cm}$ and length $22.0 \mathrm{~cm}$, from which we calculate $\alpha=2.85^{\circ}$ and $L=47.0 \mathrm{~cm}$. The working liquid was ethanol of density $\rho=810 \mathrm{~kg} \mathrm{~m}^{-3}$ and kinematic viscosity $\nu=1.52 \times 10^{-6} \mathrm{~m}^{2} \mathrm{~s}^{-1}$. The value of gravity at Azcapotzalco where the experiments were conducted is $g=9.779 \mathrm{~m} \mathrm{~s}^{-2}$.

Using silicone rubber cement, the tube was securely mounted on top of a horizontal clear acrylic plate $1 \mathrm{~cm}$ thick, the tube being aligned with a hole of diameter $D_{1}$ drilled in the plate centre. A balloon inflated to near bursting pressed against the bottom surface of the plate using a scissor jack. After pouring liquid into the cone from above, application of pressure by the scissor jack forced the balloon to spread evenly over the bottom plate surface. The experiment was initiated by pricking the balloon with a sharp object.

One side of the glass cone was marked at $1 \mathrm{~cm}$ intervals with an indelible black ink pen. The movement of the upper free surface was recorded with a Red Lake model HG-100K/HG-LE high-speed camera at 500 frames per second. Experiments showed that the time for balloon rupture is less than $0.002 \mathrm{~s}$. The location of the bottom of the curved ethanol interface was measured at selected times, interpolating to the nearest $1 \mathrm{~mm}$ between the $1 \mathrm{~cm}$ marks. The initial time was taken to be that for 


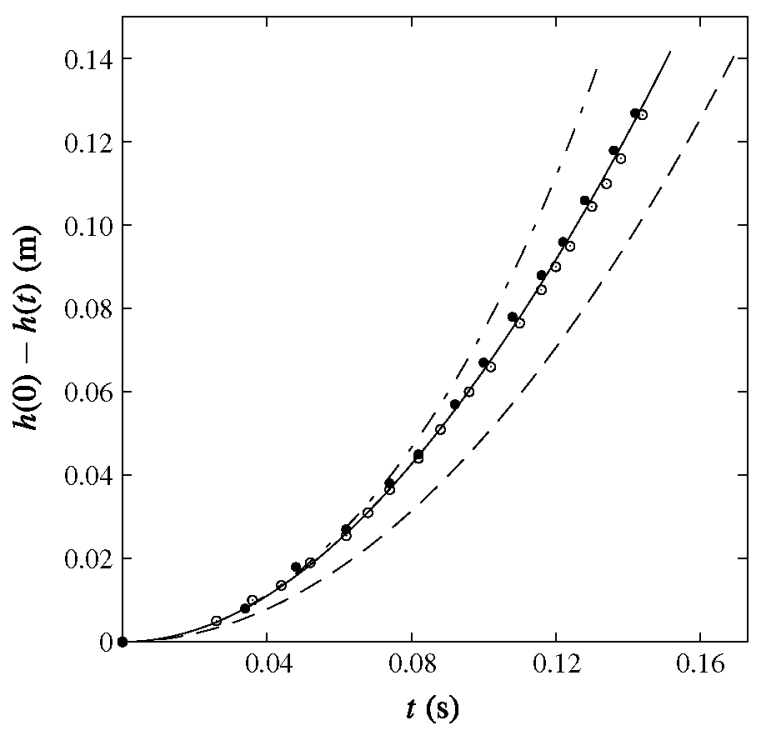

FIGURE 7. Experimental data for the interface trajectory at $\xi_{0}=0.7$ are compared with the trajectories obtained from integration of (2.10) with (solid line) and without (dot-dashed line) the VAT acceleration. The trajectory for pure free fall is shown as the dashed line. The experimental data measured by two of the present authors (A.T. and P.D.W.) are shown by the solid and open circles, respectively.

which the data best fit theory. Figure 7 shows a comparison of measurements obtained from the same video by different investigators with theoretical trajectories computed using (2.10) with (solid line) and without (dot-dashed line) the VAT acceleration term $-2 k h \dot{h}^{2}$. The results are also compared with the trajectory obtained for pure gravity given by the dashed line. While the trajectories with and without the VAT acceleration term are in good agreement up to $t \approx 0.06 \mathrm{~s}$, the results diverge considerably at late times as indicated in figure 7.

\section{Discussion and conclusion}

The theoretical model developed here corrects a peccadillo in the work of Villermaux \& Pomeau (2010) who used equation (1.1) of Paterson (1983), which neglects the VAT acceleration. The generalized Paterson equation (1.3) presented here applies to potential flow down a vertical tube when the upper free surface traverses gradually varying tube sections, be they expanding or contracting. The interface trajectories for an inverted cone exhibit qualitative differences when the VAT acceleration is retained: trajectories with (without) the VAT acceleration for a cone evolve more slowly (rapidly) than trajectories obtained where the acceleration is uniform, fixed at its initial super-accelerated value $\ddot{h}(0)=-g /(1-\beta)$.

Experiments on the evolution of free surface trajectories in inverted cones verified the formation of a nipple on the liquid interface. The results displayed in figure 7 comparing trajectories obtained from (1.1) and (1.3) for a cone show that inclusion of the VAT acceleration gives results in essential agreement with experiment. Fortunately, the oversight of Villermaux \& Pomeau (2010) in applying Paterson's equation to an inverted cone does not affect the fundamental results they report, only the details of the time evolution of the free surface. Thus their results for the initial pressure 
gradient at, and acceleration of, the upper free surface remain correct, as does the elegant proof that the initial acceleration of the centre of mass is less (in absolute value) than gravity. Moreover, their analysis of the Rayleigh-Taylor instability of the upper free surface and the evolution to drop formation also remains intact.

In addition to the parametric study obtained from integration of the generalized Paterson equation for a cone given in figures 1-3, the new results presented in this study are: (i) a determination of the singular solution for $\xi_{0}=0$; (ii) analysis of the centre-of-mass motion for tubes of arbitrary slowly varying cross-section; (iii) analysis of the terminal accelerations of the liquid interface and the centre of mass showing that they both tend to $-g$ as $t \rightarrow t_{f}$; and (iv) a comparison of the effects of interface motion with and without the VAT acceleration.

\section{Acknowledgement}

FJH acknowledges support from projects DPI2010-20450-C03-01 (Plan Nacional) and S2009/ENE-1597 (Comunidad de Madrid).

\section{REFERENCES}

Batchelor, G. K. 1967 An Introduction to Fluid Dynamics. Cambridge University Press. Paterson, A. R. 1983 A First Course in Fluid Dynamics. Cambridge University Press. Villermaux, E. \& PomeaU, Y. 2010 Super free fall. J. Fluid Mech. 642, 147-157. 